\title{
Evaluation of a Surface Exploration Traverse Analysis and Navigation Tool
}

\author{
Andrea L. Gilkey, ${ }^{1}$ Raquel Galvan ${ }^{1}$, Aaron W. Johnson, ${ }^{2}$ Ryan L. Kobrick ${ }^{3}$, Jeffrey A. Hoffman ${ }^{4}$ \\ Aeronautics \& Astronautics, Massachusetts Institute of Technology, Cambridge, MA, 02139 \\ Paulo L. Melo ${ }^{5}$ \\ MIT-Portugal Program, Massachusetts Institute of Technology, Cambridge, MA, 02139 \\ and \\ Dava J. Newman ${ }^{6}$ \\ Aeronautics \& Astronautics, Massachusetts Institute of Technology, Cambridge, MA, 02139
}

\begin{abstract}
SEXTANT is an extravehicular activity (EVA) mission planner tool developed in MATLAB, which computes the most efficient path between waypoints across a planetary surface. The traverse efficiency can be optimized around path distance, time, or explorer energy consumption. The user can select waypoints and the time spent at each, and can visualize a 3D map of the optimal path. Once the optimal path is generated, the thermal load on suited astronauts or solar power generation of rovers is displayed, along with the total traverse time and distance traveled. A field study was conducted at the Mars Desert Research Station (MDRS) in Utah to see if there was a statistical difference between the SEXTANT-determined energy consumption, time, or distance of EVA traverses and the actual output values. Actual traverse time was significantly longer than SEXTANTpredicted EVA traverse time $(n=6, p<0.01)$, traverse distance was not significantly different than SEXTANT-predicted distance, and explorer energy consumption was significantly greater than SEXTANT-predicted energy consumption $(n=5, p<0.01)$. A second study was done to see if mission re-planning, or contingency planning, was faster and less work when using SEXTANT in the habitat or in the field using an iPad. Time and workload measurements were collected for each subject under both conditions. Contingency planning in the habitat was not significantly different than contingency planning in the field. There was no significant workload difference when contingency planning in either location, however there was a trend that suggested contingency planning was faster in the habitat $(n=3, p=0.07)$. Every subject commented that it was a hassle to carry the mission planner in the field and it was difficult to see the screen in the sunlight. To determine if gloves were a factor in the difference between mission re-planning time, subjects were asked to plan a contingency indoors with and without gloves. Performance and workload were not significantly different when re-planning with and without the gloves. The SEXTANT mission planner will continue to be improved according to the results and the recommendations of subjects in this study.
\end{abstract}

\footnotetext{
${ }^{1}$ M.S. Candidate, Aeronautics \& Astronautics, 77 Massachusetts Avenue, 37-219, Cambridge, MA 02139, AIAA Student Member.

${ }^{2}$ Ph.D. Candidate, Aeronautics \& Astronautics, 77 Massachusetts Avenue, 37-219, Cambridge, MA 02139, AIAA Student Member.

${ }^{3}$ Postdoctoral Associate, Aeronautics \& Astronautics, 77 Massachusetts Ave, 37-33, Cambridge, MA 02139, AIAA Member.

${ }^{4}$ Professor of the Practice, Aeronautics \& Astronautics, 77 Massachusetts Avenue, 37-227, Cambridge, MA 02139, AIAA Member.

${ }^{5} \mathrm{Ph} . \mathrm{D}$. Candidate, MIT-Portugal Program, 77 Massachusetts Ave., E40-221, Cambridge, MA 02139.

${ }^{6}$ Professor, Aeronautics \& Astronautics, 77 Massachusetts Avenue, 33-307, Cambridge, MA 02139, AIAA Associate Fellow.
} 


\section{Nomenclature}

$\begin{array}{ll}\text { EVA } & =\text { extravehicular activity } \\ \text { GIS } & =\text { geographical information system } \\ \text { GPS } & =\text { global positioning system } \\ \text { LASOIS } & =\text { lunar astronaut spatial orientation and information system } \\ M D R S & =\text { Mars Desert Research Station } \\ M I T & =\text { Massachusetts Institute of Technology } \\ \text { SEXTANT } & \text { Surface Exploration Traverse Analysis and Navigation Tool } \\ V N C & =\text { virtual network computing }\end{array}$

\section{Introduction}

$\mathrm{F}$ OR future human missions to the Moon and Mars, efficient extravehicular activity (EVA) traverses are vitally important because resources are limited and must be used optimally. As the second EVA of Apollo 14 demonstrates, navigation in the lunar environment is challenging. ${ }^{1}$ Astronauts Al Shepard and Ed Mitchell had difficulty identifying craters and judging distances on their EVA. This led to poor situational awareness and the astronauts never reached their desired destination. To improve situational awareness while on an EVA, absolute orientation in the planetary environment is vitally important. Researchers at the Ohio State University have developed the Lunar Astronaut Spatial Orientation and Information System (LASOIS), which uses microelectromechanical systems inertial measurement units, lightweight stereo cameras, step sensors, and displays to accomplish spatial localization with the aid of an imaging system which tracks terrain targets. ${ }^{2,3}$

To further mitigate some of the challenges experienced on EVAs during the Apollo program and plan for future human planetary exploration, graduate students at the Massachusetts Institute of Technology (MIT) Man-Vehicle Laboratory have developed an EVA traverse planning tool. The first iteration of the EVA mission planner was called the Geologic Traverse Planner. ${ }^{1,4}$ The Geologic Traverse Planner featured a digital elevation map where users could select waypoints along a traverse. The mission planner then generated a straight-line path between the waypoints and output a total exploration cost, which was a measurement of metabolic cost and thermal cost. The Geologic Traverse Planner also indicated when the user violated any of the following constraints: maximum slope, maximum energy consumption, EVA time, and EVA duration.

Marquez developed informational and functional requirements for the mission planner by analyzing Apollo EVAs and field studies at the Mars Society's Haughton-Mars Project on Devon Island. ${ }^{5-8}$ Marquez overlaid the planned traverse on the terrain map, and made the metrics of time, distance, and energy cost clearly displayed on the interface, along with the elevation and slope along the traverse.

Lindqvist expanded the mission planner from a research tool to a planner that could be used for future human planetary missions by combining the mission planner program with an ArcGIS geographical information system (GIS) software program. ${ }^{9}$ Essenburg combined the two separate programs into one program written in MATLAB, call Pathmaster. ${ }^{10}$ Pathmaster also allows for mission planning with multiple explorers on the same map.

The EVA traverse planning tool used in this study is called SEXTANT, the Surface Exploration Traverse Analysis and Navigation Tool. ${ }^{11}$ SEXTANT allows users to specify waypoints on a $3 \mathrm{D}$ map of the lunar or planetary surface for an explorer to travel. Explorers can be astronauts, astronauts on transportation rovers, or unmanned robots. ${ }^{11}$ SEXTANT then calculates the optimal path between waypoints around one of three cost functions: path distance, EVA traversal time, or explorer energy consumption. The optimal path is then generated on a 3D mapping interface, and the predicted energy output is displayed, along with the total predicted traverse time and distance traveled. Crew members can use SEXTANT in the habitat to plan missions, or in the field for mission contingency planning (Figure 1).

In previous field studies of SEXTANT at the Jet Propulsion Lab and Haughton Crater, the mission planner was used by engineering students at MIT and the re-planned missions were

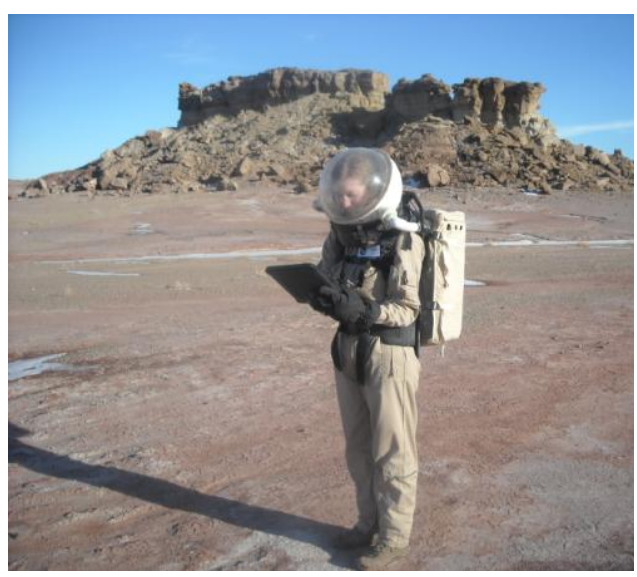

Figure 1. Mission Planning in the Field with an iPad 


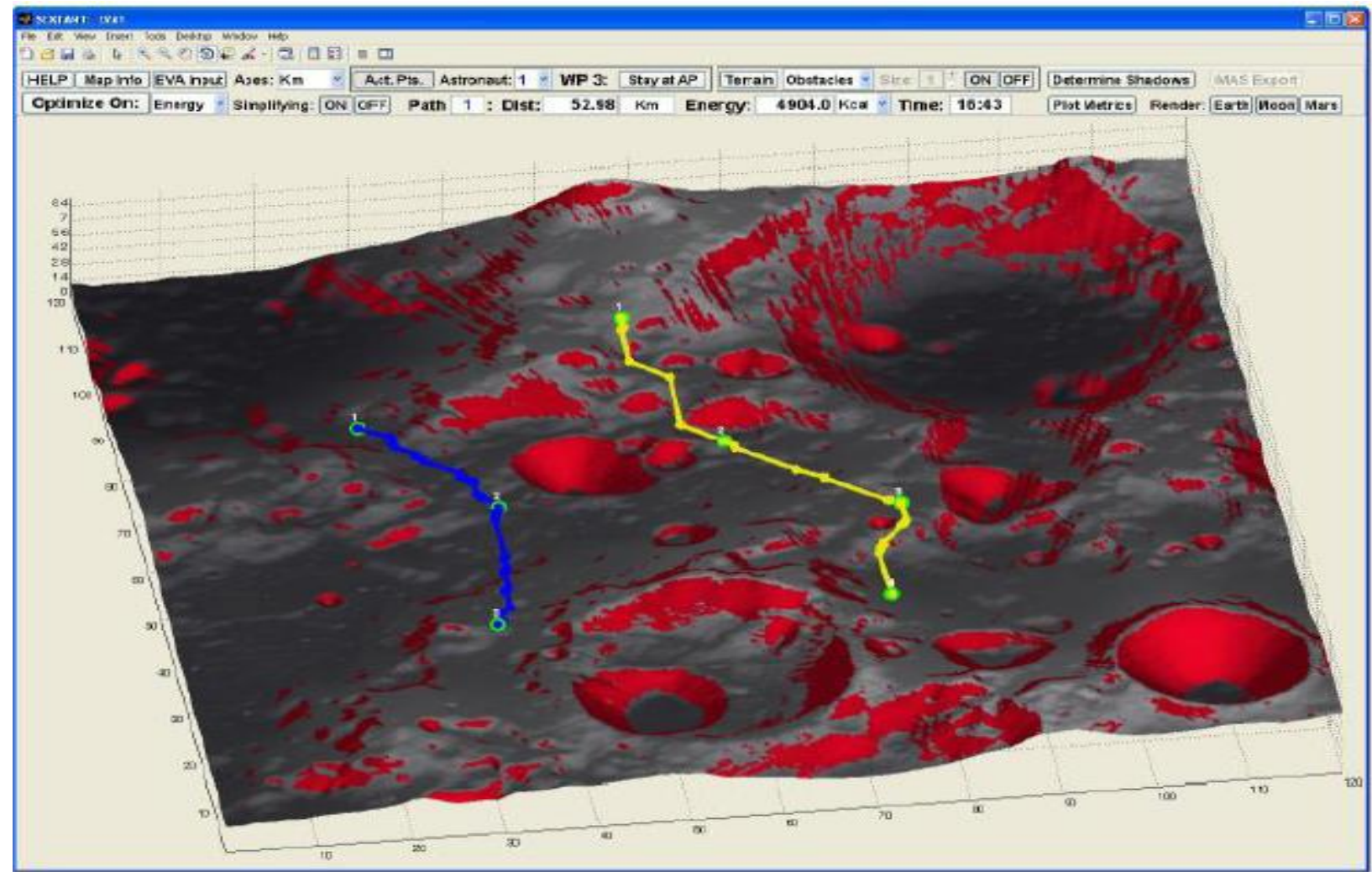

Figure 2. SEXTANT 3D mapping interface

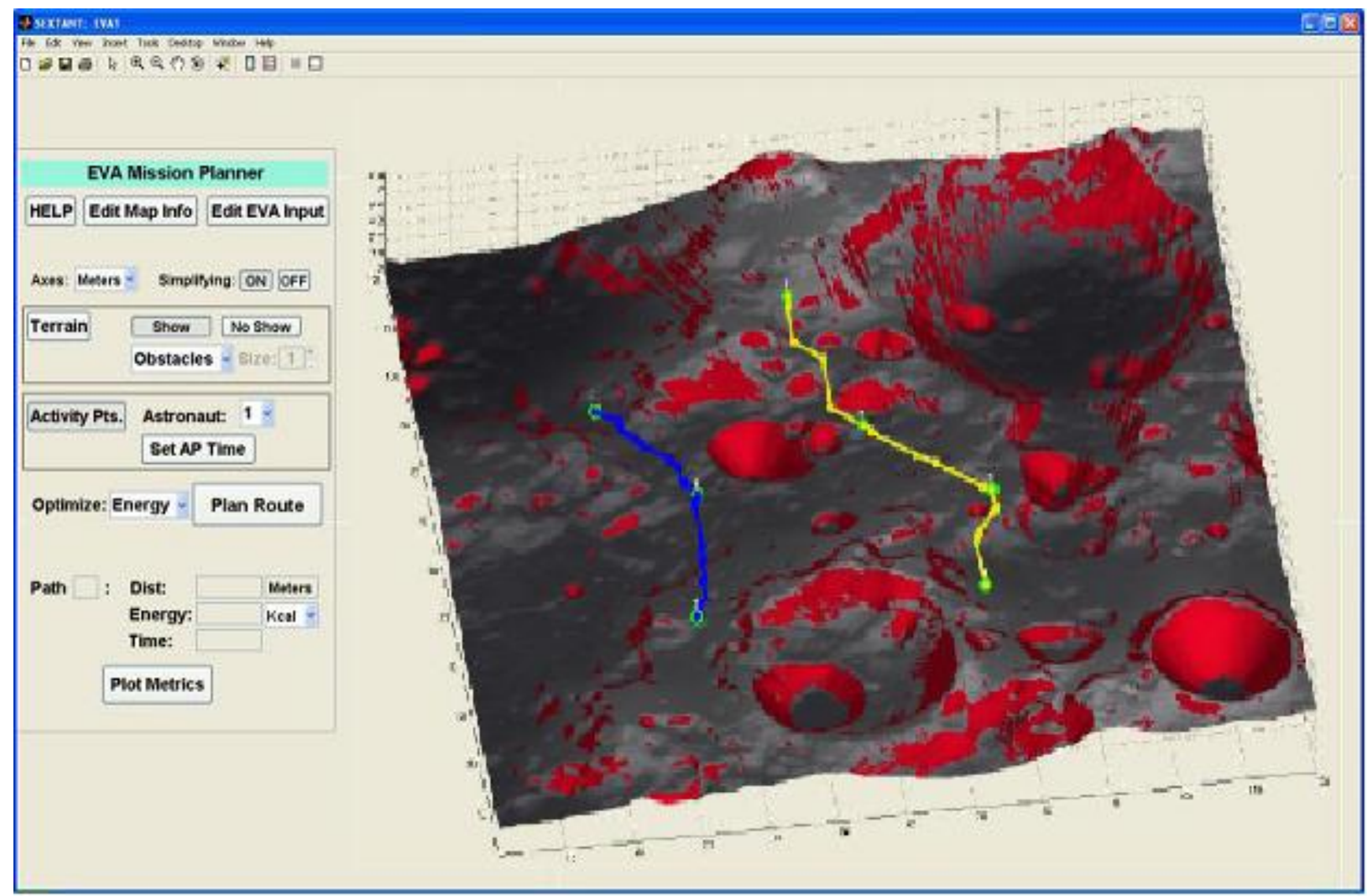

Figure 3. SEXTANT 2.0 3D mapping interface

American Institute of Aeronautics and Astronautics 
communicated to the crew members in the field. ${ }^{12}$ A future goal of SEXTANT is to be able to use it for real-time mission contingency planning in the field by the crew members. In order to develop the mission planner from primarily an engineering tool to a planner that can be used in the field, a human factors analysis was conducted on the SEXTANT computer interface. This study aims to use the mission planner for contingency planning in the field, so that the efficacy of real-time mission re-planning by the EVA crew member can be determined. Once the SEXTANT interface redesign was complete, a field study using SEXTANT was conducted.

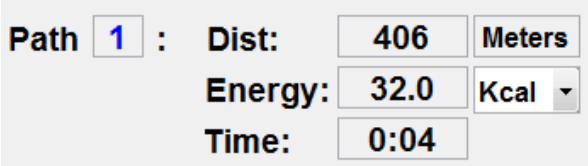

Figure 4. EVA traverse distance, subject energy consumption, and EVA time for explorer 1

\section{Materials and Methods}

At its current state, the interface of SEXTANT is designed as an engineering tool and is not designed for subject ease of use. It is desired that the interface be more user-friendly before future use in field studies. The SEXTANT interface is shown in Figure 2. In the first study, the interface was redesigned according to human factors principles. The redesigned interface is shown in Figure 3. The study consisted of 10 subjects ( 7 males, 3 females, age 21-28) who were first trained in both interfaces and then tested using each interface to perform a checklist that simulated a realistic traverse planning sequence. The total time to complete the checklist and the time to complete phases of the checklist, as well as workload ratings were obtained and analyzed statistically. Subjective feedback was also collected from each subject focusing on which interface was preferred. Within subjects analysis was done comparing performance and workload when using the SEXTANT interface and the new interface, SEXTANT 2.0.

Once the SEXTANT interface redesign was analyzed, SEXTANT was prepared for use in the field on an iPad. There are unavoidable issues with using re-planning aids in the field, such as glove interaction, weight, and visibility issues, however an iPad was chosen for this preliminary field study due to limited access to equipment. Since MATLAB cannot run natively on the iOS of an iPad, a Virtual Network Computing (VNC) system was set up between an ASUS laptop (Server) and the iPad (Client). The remote control package used for the server was TightVNC 2.0.2 set up under Windows 7 32-bit operating system and the VNC Viewer used for the client was Wyse PocketCloud $^{\mathrm{TM}}$. An ad-hoc wireless network connection was established between the laptop and the iPad and with the use of PocketCloud ${ }^{\mathrm{TM}}$. The iPad user could view and control the laptop operating system and run MATLAB, which allowed the user to view the output maps and cost functions on the iPad.

The first field study was conducted to see if there was a statistical difference between the SEXTANT-determined

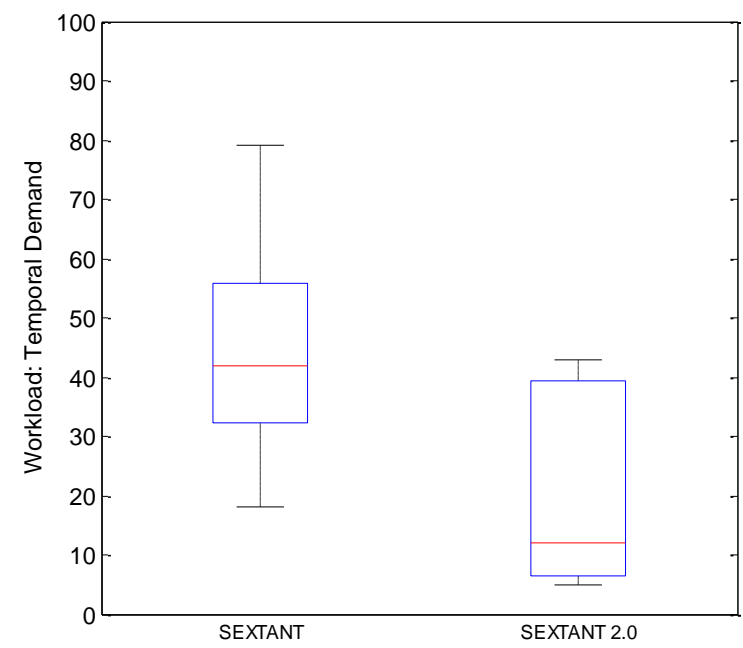

Figure 5. Workload: Temporal Demand on second trial, SEXTANT Interface vs. SEXTANT 2.0 Interface $(n=5$, $\mathrm{p}<0.05$ )

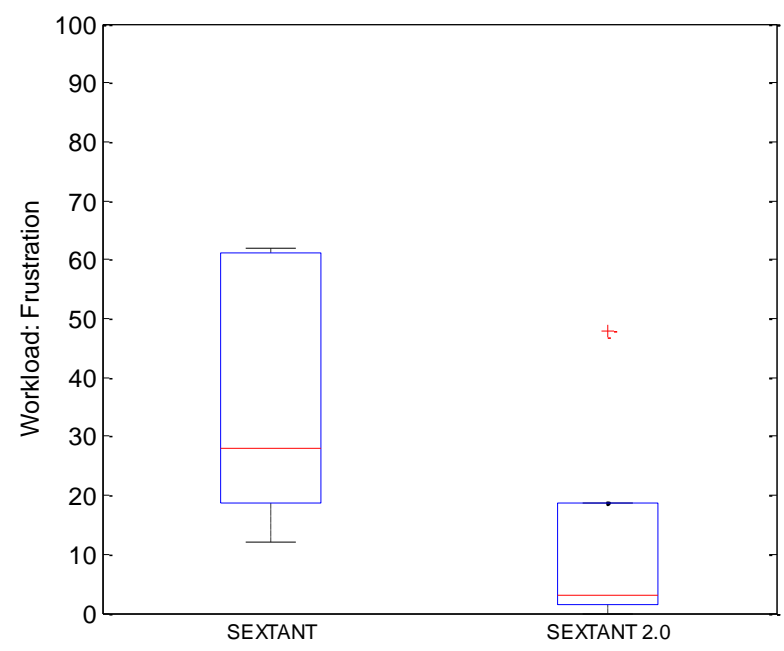

Figure 6. Workload: Frustration in second trial, SEXTANT Interface vs. SEXTANT 2.0 Interface $(n=5$, $\mathrm{p}=0.06$ ) 


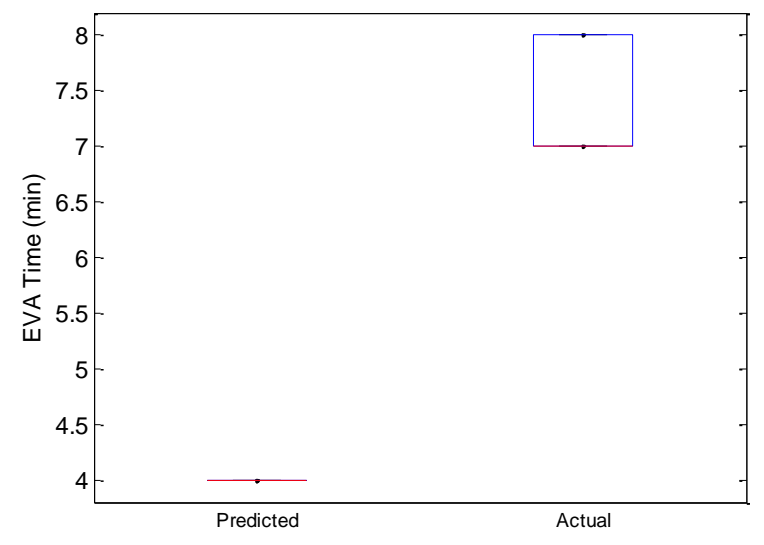

Figure 7. EVA Predicted Time vs. EVA Actual Time $(\mathrm{n}=6, \mathrm{p}<0.01)$

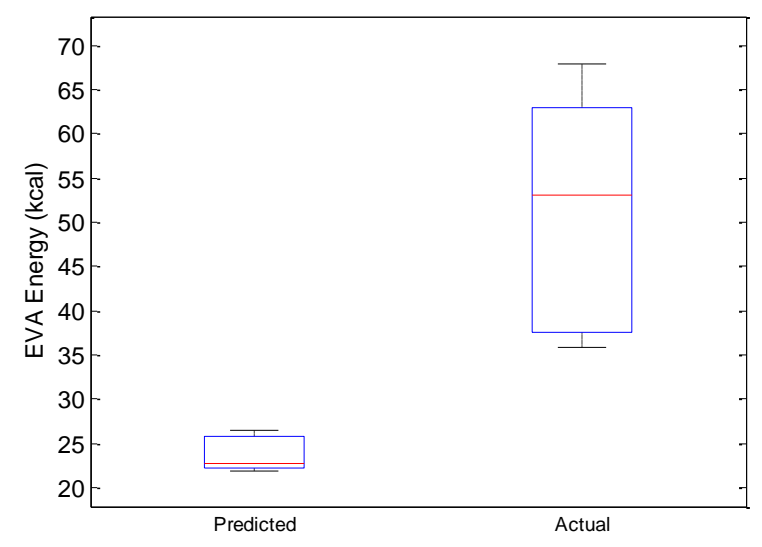

Figure 8. EVA Predicted Energy Consumption vs. EVA Actual Energy Consumption $(n=5, p<0.01)$ energy consumption, time, or distance of EVA traversals and the actual output values. The output values were displayed on the interface as shown in Figure 4. Energy consumption was determined by measuring astronaut mass, height, and heart rate along the traverse, and calculating energy consumption with energy equations. ${ }^{13}$ SEXTANT-predicted energy consumption is a function of energy rate and time. Energy rate is a function of mass, slope, gravity, and velocity. The velocity is a function of terrain slope, and is fixed depending on which slope range the terrain fits. ${ }^{11}$ The distance traveled was measured using a global positioning system (GPS). The time was measured using a stopwatch. Long EVA traversals were strenuous for the crew member subjects due to the added weight of the life support system backpack, therefore SEXTANT-predicted EVA traversals were limited to $406 \mathrm{~m}$ in length to reduce variability in crew member fitness level and to reduce stress on the subjects. There were six subjects (3 males, 3 females, ages 21-23) who completed two traverses each.

The second field study was done to see if mission re-planning, or contingency planning, was faster and less work when using SEXTANT in the habitat or in the field with an iPad. In both cases, subjects simulated adding an obstacle, such as a boulder, that blocked the traverse path and subjects had to re-plan the traverse around the obstacle. Time and workload measurements were collected for each subject under both conditions. The subjective NASA TLX workload assessment was used to determine workload. There were three subjects ( 2 males, 1 female, ages 21-23) who performed one mission re-plan in the field and one mission re-plan in the habitat, alternating which subjects re-planned in the habitat on their first trial or in the field.

The traverses started at the habitat and ended at the same location for all subjects, therefore predicted distance was the same for all trials. All traversals were conducted between the hours of 9am and 4pm MST, therefore there was adequate sunlight for each EVA traversal and subjects were performing during normal working hours. Each subject was rested according to their personal preference the night prior to testing. All subjects were comfortably fitted with the simulated space suits with no suit problems or discomforts mentioned during traversal. Each subject was weighed prior to their EVA traverse.

Subjects wore iTouch gloves in the field to re-plan missions. To ensure that the gloves alone were not making a difference in mission re-planning in the habitat and in the field, different subjects were asked post-field study to re-plan a mission both with the gloves and without the gloves. Four subjects (1 male, 3 females, ages 22-26) were asked to perform the experiment. A within subjects study was conducted, alternating if subjects wore the gloves in their first trial or not. Total time to re-plan a mission was measured, along with a NASA TLX workload survey after each trial.

Two-tailed dependent $\mathrm{t}$-tests were conducted on all studies to compare the means of several variables assuming equal variance. Nonparametric Friedman and Quade tests were performed on workload results.
Table 1. Difference in Time (sec) when Contingency Planning in the Habitat vs. in the Field

\begin{tabular}{|c|c|}
\hline $\begin{array}{c}\text { Habitat } \\
\text { (sec) }\end{array}$ & $\begin{array}{c}\text { Field } \\
\text { (sec) }\end{array}$ \\
\hline 153 & 349 \\
\hline 210 & 573 \\
\hline 221 & 369 \\
\hline
\end{tabular}




\section{Results}

\section{A. Human Factors Interface Analysis}

In the human factors analysis of the SEXTANT interface redesign, learning was the largest contributing factor to the results. Subjects were significantly faster at planning missions on the second trial, regardless of which interface the subject used first $(n=10, p<0.05)$. It was found that with the new interface, SEXTANT 2.0, marginally less time was spent inputting explorer properties and the EVA schedule $(n=10, p=0.07)$. When looking at workload factors, there was marginally less workload for two of the six workload categories when using SEXTANT 2.0: mental demand $(\mathrm{n}=10, \mathrm{p}=0.06)$ and frustration $(\mathrm{n}=10, \mathrm{p}=0.09)$.

Because learning was involved, workload measurements were also compared between subjects using SEXTANT and SEXTANT 2.0 on the second trial. When comparing the workload on the five subjects that used SEXTANT second to the five subjects that used SEXTANT 2.0 second, it was found that temporal demand was significantly less with SEXTANT $2.0(\mathrm{n}=5, \mathrm{p}<0.05)$, and that frustration was marginally less with SEXTANT $2.0(n=5, p=0.06)$. The box plots of temporal demand and frustration for SEXTANT vs. SEXTANT 2.0 are shown in Figure 5 and Figure 6, respectively.

Although overall there was no strong quantitative difference between the two interfaces, 8 out of 10 subjects preferred SEXTANT 2.0 over SEXTANT. Because of the results of this study, SEXTANT 2.0 was used in the MDRS field studies.

\section{B. Mission Planning}

The difference in traversal time, traversal distance, and explorer energy consumption between SEXTANT and actual values was measured. Actual EVA traverse time was significantly different than SEXTANTpredicted time $(n=6, p<0.01)$, as shown in Figure 7 . Actual traverse distance was not significantly different than the predicted EVA traverse distance. Explorer energy consumption was significantly different than the predicted energy consumption $(n=5, p<0.01)$, as shown in Figure 8. Each of the six subjects commented that it was difficult to carry the mission planner in the field and it was difficult to see the screen in the sunlight, especially with the space suit helmet on.

The ability to plan a contingency during EVA is vitally important in cases of emergency. If a crew member were to experience a problem with the space suit, a mission planner would have the ability to ensure a quick and reliable return to the habitat. To compare mission re-planning inside the habitat with contingency planning in the field using an iPad, time and workload measurements were collected for each subject under both conditions. The times it took to re-plan in the habitat and in the field are shown in Table 1. Contingency planning in the habitat was not significantly less than contingency planning in the field, however there was a trend $(n=3, p=0.07)$, as shown in Figure 9. There was no significant workload difference when re-planning in the habitat or in the field.

When subjects re-planned a mission in the habitat, they used the iPad but they were not wearing the iTouch gloves. To see if wearing the iTouch gloves made a difference in the time spent re-planning a mission, subjects inside were asked to re-plan a mission wearing the gloves and without wearing the gloves. There was not a significant difference between the total time subjects spent re-planning a mission when wearing the gloves or wearing no gloves, however there was a trend towards better performance without gloves $(n=4, p<0.1)$, as shown in Figure 10. There was a trend in three of the six workload ratings (perceived performance, effort, and frustration) that

$$
6
$$

American Institute of Aeronautics and Astronautics 
showed that workload was less when re-planning a mission without the gloves. Subjects thought they performed better without the gloves ( $n=4, p=0.06$, Figure 11), used less effort $(n=4, p=0.09$, Figure 12), and were less frustrated $(\mathrm{n}=4, \mathrm{p}=0.09)$ than when they were wearing the gloves.

Additionally, a between subjects comparison was made with the subjects that performed mission re-planning inside with gloves and the subjects that performed mission re-planning in the field with gloves. There was not a significant difference between the mission re-planning time of subjects inside wearing gloves and in the field, however there was a trend that suggested that mission re-planning inside wearing gloves was faster than mission replanning in the field $(n=7, p=0.07)$, suggesting that additional factors other than gloves alone led to the difference in planning time in the field and in the habitat.

\section{Discussion}

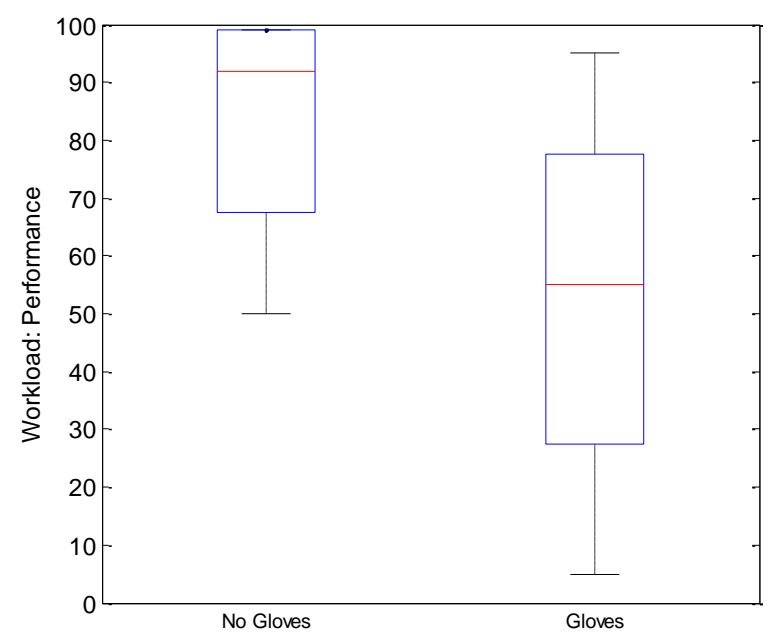

Figure 11. Workload: Performance Rating when Contingency Planning Without Gloves vs. With Gloves $(n=4, p=0.06)$

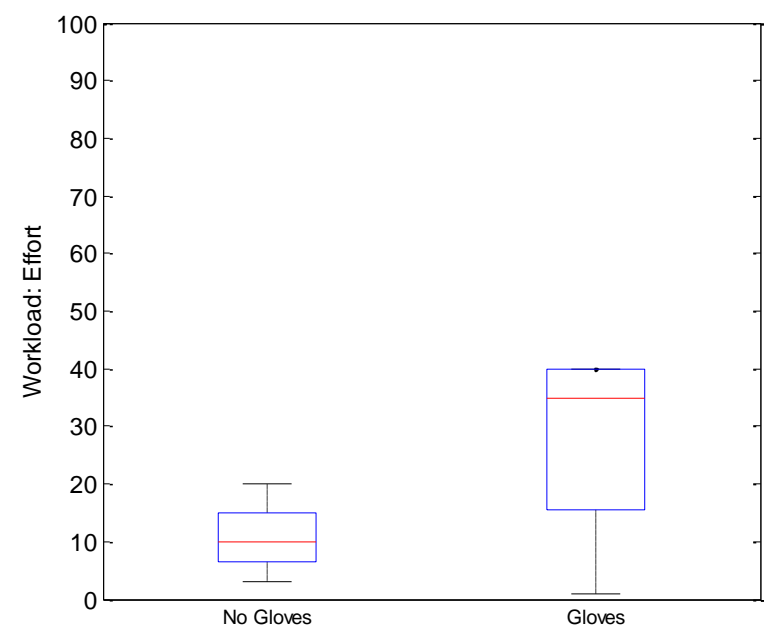

Figure 12. Workload: Effort Rating when Contingency Planning Without Gloves vs. With Gloves $(n=4, p=0.09)$
There was a significant difference in subject energy consumption between SEXTANT-predicted energy consumption and actual energy output. The equations used to calculate energy consumption take into account actual EVA traverse time, while SEXTANT uses predicted EVA traverse time. ${ }^{14}$ Because energy expenditure calculations used actual traverse time, this could be a reason for the significant difference in crew member energy consumption between actual energy expenditure and SEXTANT-predicted energy expenditure.

Heart rate was monitored at the beginning of the traverse, the end of the traverse, and every three minutes during testing. Heart rates were determined between these times through interpolation instead of through continuous measurements. Average heart rate (in beats per minute) was calculated for each traverse and input into the energy equations. Discrete heart rate data points could have also led to a difference in actual energy consumption values, however the terrain over which the traverses took place was relatively flat, with no large slopes or hills; therefore the heart rate should not have changed by large amounts between heart rate measurement points.

An iPad was selected as the mission planning tool used in the field study due to limited access to equipment. Although there may be human factors issues associated with using an iPad in the field, this was a preliminary study accessing SEXTANT and future studies will include more advanced equipment.

The data showed that there was no significant difference between mission re-planning in the field and in the habitat, however there was a trend that suggested that EVA re-planning was faster in the habitat. Each of the six subjects mentioned that it was difficult to see the iPad screen when re-planning a mission in the field due to bright sunlight, which indicates sunlight may have been a factor contributing to this difference. Also, when subjects re-planned the mission in the field they wore gloves to protect against the harsh environment of analog Mars. Gloves were not worn when re-planning in the habitat. The data from the follow-up study at MIT showed that there was not a significant difference 
between mission planning without gloves and with the gloves, however this also showed a trend that may have indicated otherwise if more subjects participated. These results indicate that perhaps gloves as well as the bright sunlight may contribute to mission re-planning time in the field and both factors should be taken into account on future planetary missions. Future re-planning tools should be less weight than an iPad, easily compatible with gloves, and allow for viewing even in direct sunlight.

Significant differences were only seen between SEXTANT-predicted energy expenditure and time calculations and the actual energy and time measurements. All other observations were not significant or only demonstrated a trend in the data. A limitation of this field study is subject size. Only six subjects were at MDRS, therefore this was the maximum subject number possible for the field studies. All six subjects were used to compare SEXTANTpredicted EVA traverse time, distance, and subject energy consumption to actual values. The length of stay at MDRS was two-weeks long, and each of the six crew members had individual projects to conduct during the stay, therefore subject number and availability, as well as field testing time availability was limited. Nonparametric statistics tests have little power when sample size is small. The statistics could be strengthened with a larger number of subjects.

If this study were to be repeated, recommendations include conducting repeated EVAs to various locations, some closer to the habitat, and some further away from the habitat. The EVA destinations would also include sloped terrain, with hills and drops similar to craters to measure SEXTANT algorithms over a variety of terrain features. The crew member heart rate would also be measured continuously on future studies. Also, this study would be greatly improved with a larger sample size, therefore a future field study would include several subjects instead of three to six.

For future human missions to the Moon, Mars, and beyond, an EVA mission planner is extremely important. By effectively predicting EVA costs such as traverse distance, time, and explorer energy consumption, astronauts, engineers, and mission control can ensure that the crew will not exceed life support system consumables and will take the optimal path to reach their desired destinations. A mission planner that determines these variables and allows for contingency planning in the field is an important step in preparing humans for further space exploration.

\section{Conclusion}

The SEXTANT 2.0 interface showed a trend towards improved user performance and reduced workload over the SEXTANT interface, with qualitative support from subjects. The SEXTANT 2.0 interface was used on field studies at MDRS, which found that SEXTANT-predicted EVA traversal time and subject energy consumption were significantly different than actual time and energy consumption, while there was no significant difference in EVA distance from SEXTANT-predicted values. Contingency planning showed a trend towards improved performance when re-planning in the habitat compared to in the field, however workload was not significantly different. Finally, there was not a significant difference between mission planning performance and workload without gloves and with gloves, however results indicated a trend that suggested mission planning was better in terms of performance and workload when subjects were not wearing gloves. The results of this study will be used to further improve characteristics of SEXTANT.

\section{Acknowledgments}

This field study was made possible by grants from the NASA Astrobiology Institute and Massachusetts Space Grant. Thank you to the Mars Society for allowing this field study to be conducted at the Mars Desert Research Station.

\section{References}

${ }^{1}$ Carr, C. E., Newman, D. J., and Hodges, K., "Geologic Traverse Planning for Planetary EVA”, SAE 2003-01-2416, 33rd International Conference on Environmental Systems, Vancouver, BC, Canada, 7-10 July 2003.

${ }^{2}$ Li R, Di K, Wu B, Yilmaz A, Banks MS, Oman C, Bhasin K, Tang M. (2008) Enhancement of spatial orientation capability of astronauts on the lunar surface supported by integrated sensor network and information technology. NLSI lunar science conference, Moffett Field, CA.

${ }^{3}$ Li R, Wu B, He S, Shopljak B, Yilmaz A, Jiang J, Banks MS, Oman C, Bhasin KB, Warner JD, Knoblock EJ (2009) LASOIS: enhancing the spatial orientation capabilities of astronauts on the lunar surface. In: Proceedings of the $40^{\text {th }}$ lunar and planetary science conference, The Woodlands, TX.

${ }^{4}$ Carr, C. E., Distributed Architectures for Mars Surface Exploration, M.S. thesis, Massachusetts Institute of Technology, 2001. 
${ }^{5}$ Márquez, J. J., Human-Automation Collaboration: Decision Support for Lunar and Planetary Exploration, Ph.D. thesis, Massachusetts Institute of Technology, 2007.

${ }^{6}$ Márquez, J. J., and Cummings, M.L., "Design and Evaluation of Path Planning Decision Support for Planetary Surface Exploration", Journal of Aerospace Computing, Information, and Communication, 5(5), 2008, pp. 57-71.

${ }^{7}$ Márquez, J. J., and Newman, D. J., "Planning and Re-planning for Planetary Extravehicular Activities: Analysis of Excursions in a Mars-Analog Environment and Apollo Program", SAE 2006-01-2297, $36^{\text {th }}$ International Conference on Environmental Systems, Norfolk, VA, 17-20 July 2006.

${ }^{8}$ Márquez, J. J., and Newman, D. J., "Recommendations for Real-Time Decision Support Systems for Lunar and Planetary EVAs", SAE 2007-01-3089, 37th International Conference on Environmental Systems, Chicago, IL, 9-12 July 2007.

${ }^{9}$ Lindqvist, L. V. J., Multidisciplinary Extravehicular Activity Mission Optimization for Lunar Exploration, M.S. thesis, Technishe Universität München, 2008.

${ }^{10}$ Essenburg, J. R., Mission Planning and Navigation Support for Lunar and Planetary Exploration, M.S. thesis, Massachusetts Institute of Technology, 2008.

${ }^{11}$ Johnson, A.W., An Integrated Traverse Planner and Analysis Tool for Future Lunar Surface Exploration, M.S. thesis, Massachusetts Institute of Technology, 2010.

${ }^{12}$ Johnson AW, Newman DJ, Waldie JM, Hoffman JA (2009) An EVA mission planning tool based on metabolic cost optimization. International conference on environmental systems (ICES), Paper number 2009-01-2562, Savannah, GA.

${ }^{13}$ Keytel, L.R., Goedecke, J.H., Noakes, T.D., Hiiloskorpi, H., Laukkanen, R., van der Merwe, L., \& E.V. Lambert. Prediction of energy expenditure from heart rate monitoring during submaximal exercise. Journal of Sports Sciences. 23, 289-97 (2005).

${ }^{14}$ Santee, W.R., Allison, W.F., Blanchard, L.A., and Small, M.G. "A proposed model for load carriage on sloped terrain", Aviation, Space, and Environmental Medicine. 72(6), 2001. 